\title{
Closed-loop liquid-vapor equilibrium in a one-component system
}

\author{
N. G. Almarza \\ Instituto de Química-Física Rocasolano, CSIC, Serrano 119, E-28006 Madrid, Spain
}

(Received 21 June 2012; published 5 September 2012)

\begin{abstract}
We report Monte Carlo simulations that show a closed-loop liquid-vapor equilibrium in a pure substance. This finding has been achieved on a two-dimensional lattice model for patchy particles that can form network fluids. We have considered related models with a slightly different patch distribution in order to understand the features of the distribution of patches on the surface of the particles that make possible the presence of the closed-loop liquid-vapor equilibrium, and its relation to the phase diagram containing so-called empty liquids. Finally we discuss the likelihood of finding the closed-loop liquid-vapor equilibria on related models for three-dimensional models of patchy particles in the continuum, and speculate on the possible relationship between the mechanism behind the closed-loop liquid-vapor equilibrium of our simple lattice model and the salt-induced reentrant condensation found in complex systems.
\end{abstract}

DOI: 10.1103/PhysRevE.86.030101

PACS number(s): 64.10.+h, 64.60.aq, 81.16.Dn

Recent advances in the chemical synthesis of colloidal particles that are monodisperse in shape and size have generated a lot of theoretical work to understand their collective behavior [1,2]. These particles can be thought as "colloidal molecules" that can organize themselves by self-assembly into useful structures [3]. Patchy colloids [4] are very interesting because of the possibility of tuning the number, interaction, and local arrangements of their attractive sites. The structure of the self-assembled clusters of the patchy colloids therefore can produce different macroscopic behavior [1-4]. On the other hand, primitive models of patchy particles have been used to represent the specific anisotropic interactions between globular proteins in solution [4]. These systems sometimes present reentrant condensation induced by variations of salt concentration $[5,6]$. The mechanisms involved in the condensation and the dependence of the reentrant behavior with temperature are not well understood, but it is likely that specific (patchylike) interactions [7,8] play an important role.

Given the complexity of both kinds of problems, selfassembly of patchy colloids and the phase behavior of protein solutions, it seems important to study simple models of the patchy particles in order to understand the influence of the numbers of patches and their distribution on the phase behavior and aggregation mechanisms of complex systems.

Recently it has been found that patchy models with two types of patches, $A$ and $B$, in which only $A-A$ and $A-B$ interactions are attractive, can exhibit liquid-vapor equilibria (LVE) with liquid phases behaving as "empty liquids" [9-11]. For these systems the term "empty liquid" [4] refers to the fact that at low temperatures the density of the liquid phase $\rho_{L}$ on the binodal becomes very small at low temperature. Actually $\rho_{L}$ approaches zero in the limit $T \rightarrow 0$. The empty liquid phenomenology has been found for hard sphere particles that carry on the surface two patches of type $A$ located at opposite poles of the sphere, and several patches of type $B$ elsewhere on the surface. The potential energy is defined as a sum of bonding interactions between pairs of patches belonging to different particles.

Within this interaction scheme, the shape of the phase diagram basically depends on the ratio between the bonding energies of the two types of possible bonds, $y=\epsilon_{A B} / \epsilon_{A A}$. It is known from both Monte Carlo simulation and Wertheim's perturbation theory [9-12] that the regime of the empty liquid may occur for $y<1 / 2$. Theoretical approximations also predict [12] a lower limit for this ratio, namely, $y_{l}=1 / 3$. Simulation results are consistent with the existence of such a lower bound $\left(y_{l}>0\right)$ for the empty liquid regime. On the other hand, for $y=0$, the systems at low temperature are expected to form linear chains [13], which may give rise to orientational ordered (nematic) phases at moderate densities [14-16]. This orientational order can also appear as a second phase transition for values of $y<0.5$ where the LVE occurs. The topology of the empty liquid phases is well understood [9-12]. At low temperature (where the empty liquid regime occurs) and not too low density almost all the $A$ patches are participating in a bond, and the liquid phases then can be described as a network fluid composed of (almost) straight chains of spheres linked by sequences of $A A$ bonds. Each of the terminal spheres of the chains is connected to another straight chain by an $A B$ bond. In the empty liquid regime the reduction of the temperature implies an increase in the typical length of the $A A$ chains, which in turn produces a reduction in the density of the liquid phase. This is due to the fact that $A A$ bonds for $y<1 / 2$ are energetically dominant.

At this point, we can ask ourselves to what extent this physical picture remains valid if the nature of the colloid surface is changed, and the two $A$ patches are no longer located at opposite poles. We can define a bending angle $\theta_{i j k}$ for a triplet of particles $\{i, j, k\}$ in which $j$ is linked through $A A$ bonds to both $i$ and $k$ as

$$
\cos \theta_{i j k}=\frac{\vec{r}_{j i} \cdot \vec{r}_{j k}}{\left|\vec{r}_{j i}\right|\left|\vec{r}_{j k}\right|} .
$$

Taking into account that the size of the patches is supposed to be small in order to preserve the condition of only one bond per patch [9], the mean value of $\cos \theta_{i j k}$ will fulfill $\left\langle\cos \theta_{i j k}\right\rangle \simeq \cos \alpha_{A A}$, where $\alpha_{A A}$ is the angle between the vectors that connect the center of the sphere with the centers of its $A$ patches. Notice that reducing the value of $\alpha_{A A}$ from $180^{\circ}$ implies an increase in the probability of forming rings of particles connected by $A A$ bonds, since the system can reduce its energy by forming cycles. Moreover, when one of these cycles is formed all the $A$ patches of its particles are bonded, 
and therefore these patches cannot bond other chains, which in turn can reduce the connectivity of different parts of the system, and eventually inhibit the appearance of LVE.

Considering that for $\alpha_{A A}=180^{\circ}$ and appropriate values of $y$ one can find the empty liquid regime at low temperatures, and that reducing the value of $\alpha_{A A}$ might, in principle, destroy the LVE, it seems interesting to know what happens between the two scenarios. In order to investigate this issue, we have found it convenient to analyze a simple lattice model whose results are expected to be representative of what can be found for models in the continuum [11,17]. The computation of phase diagrams is usually much cheaper in terms of CPU time for lattice models, which, in spite of their simplicity, can often capture the essential features of the phase diagrams of associating molecules and network fluids [11,17-25].

We have considered models on the triangular lattice (see Fig. 1). Sites on the lattice can be either empty or occupied by one particle. Each particle carries two sites (of type $A$ ) that point to two of the nearest-neighbor (NN) sites. Two particles, $i, j$, being NNs, interact with an energy $u_{i j}=-\epsilon$ if each one points to the other with one of its $A$ patches ( $A A$ bond); if only one of the particles points to the other with one of its $A$ patches, we have $u_{i j}=-y \epsilon(A B$ bond, with $0<y \leqslant 1 / 2)$, and otherwise $u_{i j}=0$.

We have studied three models (see Fig. 1), which will be denoted as $o-, m-$, and $p$-2A4B models (by borrowing the prefixes from organic chemistry nomenclature for the positions of the substituents in aromatic cyclic compounds: ortho, meta, and para). The angle $\alpha_{A A}$ takes respectively the values $60^{\circ}$, $120^{\circ}$, and $180^{\circ}$ for the $o-2 \mathrm{~A} 4 \mathrm{~B}, m-2 \mathrm{~A} 4 \mathrm{~B}$, and $p-2 \mathrm{~A} 4 \mathrm{~B}$ models. In the $o-2 \mathrm{~A} 4 \mathrm{~B}$ model cycles of $A A$ bonds are easily formed, and the smallest possible cycle contains just three particles, and in the $m$-2A4B model we can find cycles of six $A A$ bonds, whereas in the $p-2 \mathrm{~A} 4 \mathrm{~B}$ models no cycles are possible. In order to compute the phase diagram we have made use of simulation strategies that were described previously $[11,17-$ 19], namely, Wang-Landau multicanonical simulations [11,26] to locate critical points and some liquid-vapor coexistence points, and Gibbs-Duhem integration to trace the binodals. Some special techniques have been developed in the simulation procedures to improve the sampling, including efficient cluster (a)

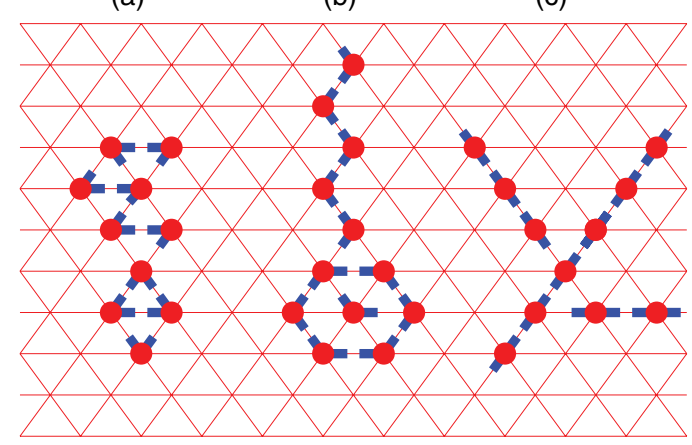

FIG. 1. (Color online) Sketch of the patchy particles in the three lattice models: (a) $o$-2A4B model, $\alpha_{A A}=60^{\circ}$; (b) $m$-2A4B model, $\alpha_{A A}=120^{\circ}$; (c) $p$-2A4B model, $\alpha_{A A}=180^{\circ}$. Thin lines represent the underlying triangular lattice. Solid circles represent particles (occupied sites). Thick segments indicate the orientation of $A$ patches.

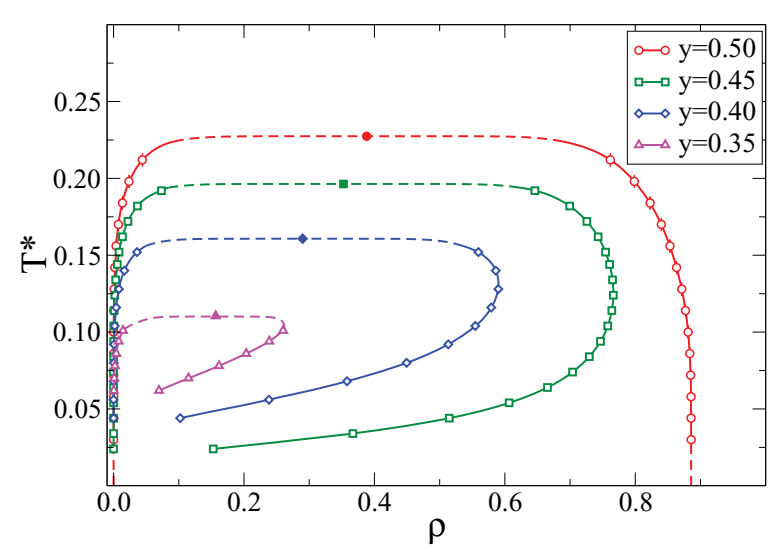

FIG. 2. (Color online) Temperature-density phase diagrams for the $p$-2A4B model, and different values of $y$ (see the legends). Solid symbols indicate critical points. $T^{*}$ is the reduced temperature, $T^{*}=$ $k_{B} T / \epsilon_{A A}$ (where $k_{B}$ is the Boltzmann constant), and the density $\rho$ corresponds to a fraction of occupied sites.

algorithms for the $p$-2A4B model. Technical details will be published elsewhere [27].

We have not found evidence of LVE for the $o$-2A4B model. This seems plausible, since at low temperature and moderate density the particles can easily arrange themselves in triplets that minimize the energy and block the development of an extended network of patch-patch bonds. The $p$-2A4B model shows, as expected, a phase diagram (see Fig. 2) that fully resembles those of the analog models in the continuum $[9,10]$, and on the square lattice [11], i.e. the empty liquid regime appears.

The key result of this Rapid Communication is the phase diagram of the $m$-2A4B model (shown in Fig. 3). For $y=0.50$ we get a similar behavior in the $m-2 \mathrm{~A} 4 \mathrm{~B}$ and $p-2 \mathrm{~A} 4 \mathrm{~B}$ models, i.e., we find typical LVE binodals. However, in the $m$-2A4B model the LVE for $y_{l}<y<1 / 2$, with $y_{l} \simeq 0.43$, exhibits two critical points, which will be named, according to their critical temperature, as the lower critical point (LCP) and the upper critical point (UCP). The LVE only occurs between these two temperatures, which of course depend on the value of $y$. For this model the critical points were determined through a finite-size scaling analysis on the multicanonical simulation

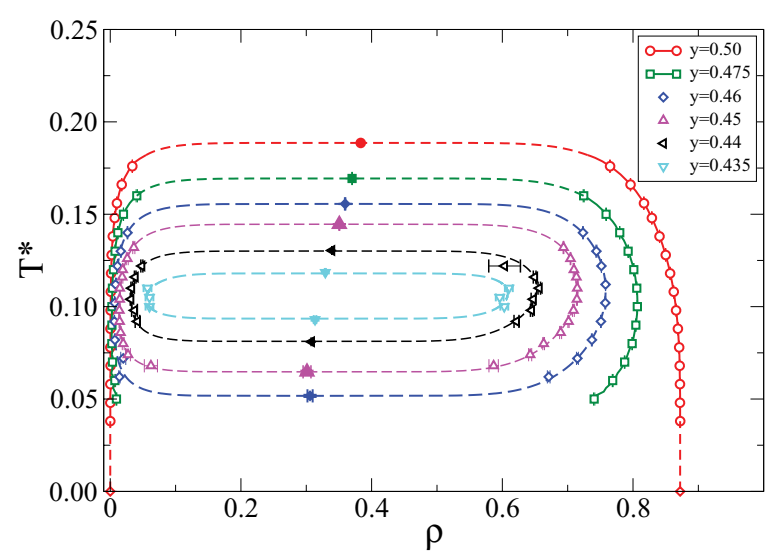

FIG. 3. (Color online) Temperature-density phase diagrams for the $m$-2A4B model. See the caption of Fig. 2 for notation details. 
[11] considering system sizes in the range $12 \leqslant L \leqslant 48$ (with $L^{2}$ being the total number of sites). The scaling behavior was found to be fully consistent with the two-dimensional Ising criticality for both LCP and UCP. The existence of reentrant phase equilibria is known to appear in some binary mixtures. For one-component systems it is very unusual to find fluid-fluid reentrant critical behavior. Roberts et al. [20] found such a behavior for a lattice model of network-forming fluids, however, the closed-loop coexistence found in Ref. [20] involves liquid phases. In our case, the phase equilibria can be appropriately described as LVE, by considering that modifying the value of the interaction parameter $y$, the reentrant phase diagrams can be connected continuously to typical LVE diagrams that appear for $y \geqslant 1 / 2$.

We have indicated above that the closed-loop LVE occurs for any value of $y$ in the range $\left(y_{l}, 1 / 2\right)$, however, our simulation results only support, in principle, the reentrance for $y_{l}<y \leqslant 0.46$. As $y$ approaches $1 / 2$, it becomes a very hard task to compute the LCP using a Monte Carlo simulation, since the LCPs occur at very low temperatures. However, we can make use of the scaling criteria introduced in Ref. [11]: At very low temperature, the probability of finding nonbonded $A$ patches becomes negligibly small, and the potential energy of the model can be approximated as [11] $(U / \epsilon) \simeq-N+\mathcal{N}_{A B}\left(\frac{1}{2}-y\right)$, where $N$ is the number of occupied sites and $\mathcal{N}_{A B}$ the number of $A B$ bonds. Therefore the phase behavior at low temperature $T$ will basically depend on the ratio $(1 / 2-y) \epsilon / k_{B} T$ (with $k_{B}$ being the Boltzmann constant). Then the temperature at the LCP is expected to scale for $(1 / 2-y) \rightarrow 0^{+}$as $T_{C}^{l} \propto(1 / 2-y)$. The low temperature phase diagram of the $m-2 \mathrm{~A} 4 \mathrm{~B}$ model has been found to fulfill very well this scaling at low temperature (see Fig. 4). Of course the reliability of this prediction depends on the absence of additional phase transitions that could occur in the model. Notice that the $p$-2A4B model is expected to exhibit an order-disorder transition [11,14-16]. In the case of the $m$-2A4B model we have not found any signature of a second phase transition at the temperature range where the close-loop LVE occurs.

The origin of the different topologies of the LVE phase diagrams of the $p$ - and $m$-2A4B models can be explained by looking at representative configurations of both models at low

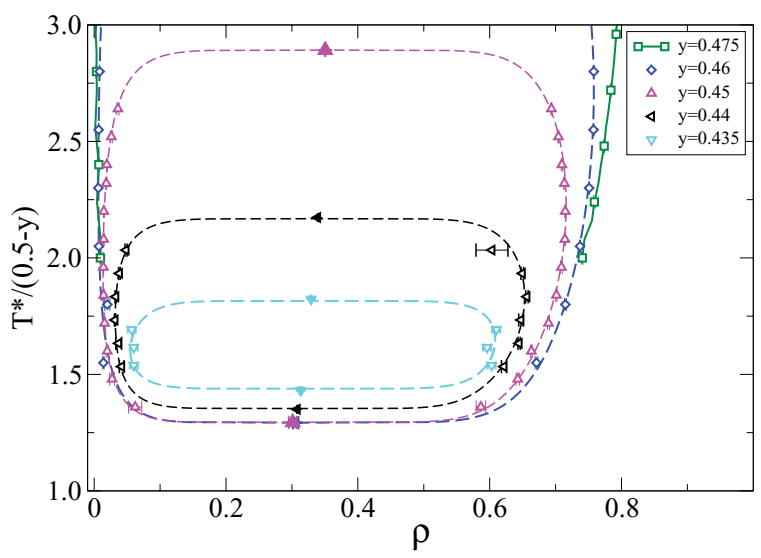

FIG. 4. (Color online) Liquid-vapor equilibria phase diagrams for the $m$-2A4B model with an alternative temperature scaling.

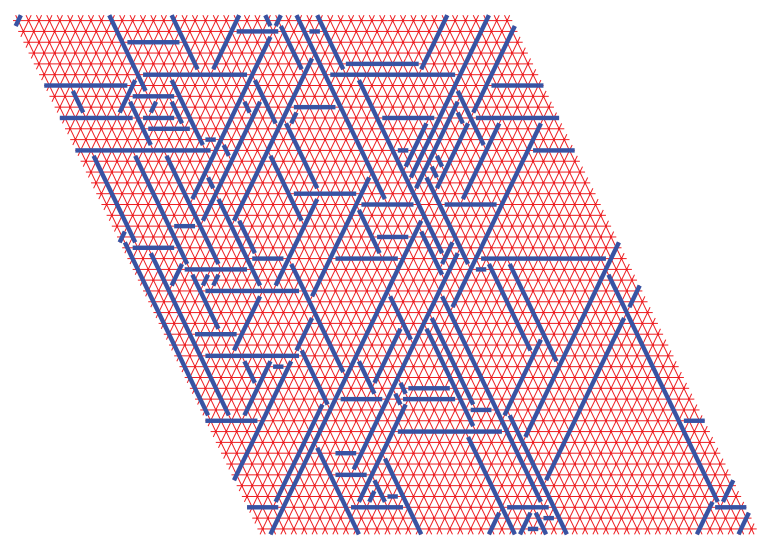

FIG. 5. (Color online) Configuration for the liquid phase at the binodal for the $p$-2A4B model with $y=0.40, T^{*}=0.07, \rho \simeq 0.375$.

temperature. In the $p$-2A4B model (Fig. 5) the end particles of each chain are bonded via $A B$ bonds to two other chains on the system, then on average each chain is bonded to four neighbor chains $\left(\mathcal{N}_{A B}=2 N_{\text {chains }}\right.$ at low $\left.T\right)$. At very low temperature in the $m-2 \mathrm{~A} 4 \mathrm{~B}$ model we can see (Fig. 6) the formation of rings with different numbers of particles, and the existence of $A B$ intrachain bonds. As a consequence the number of available $A$ patches to form interchain bonds is reduced, and the bonds do not produce a macroscopic bond network. At higher temperature (Fig. 7) there is a reduction in the number of rings due to entropic effects [28]; this enhances the connectivity and the phase separation can take place by a mechanism similar to that present in the $p-2 \mathrm{~A} 4 \mathrm{~B}$ model.

It has to be emphasized that the origin of the reentrance of the LVE is not just the angle between patches in the colloidal particles, but the fact that at low temperature the particles have the trend to self-assemble, forming small aggregates (usually rings) in which most of their sticky spots are saturated by intra-aggregate interactions. This process reduces the effective valence of the aggregates with respect to that of the independent particles, which in turn reduces the possibility of bond network formation and the ability of the system to condense.

The mechanism underlying the reentrance of the LVE is rather different from that of binary liquid mixtures, in which

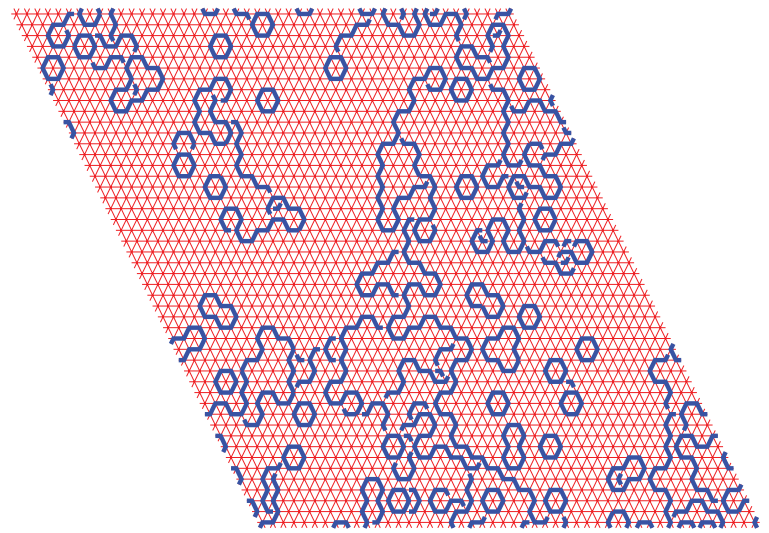

FIG. 6. (Color online) Configuration for the $m$-2A4B model with $y=0.44, \rho=1 / 3, T^{*}=0.03$ (below the lower critical temperature). 


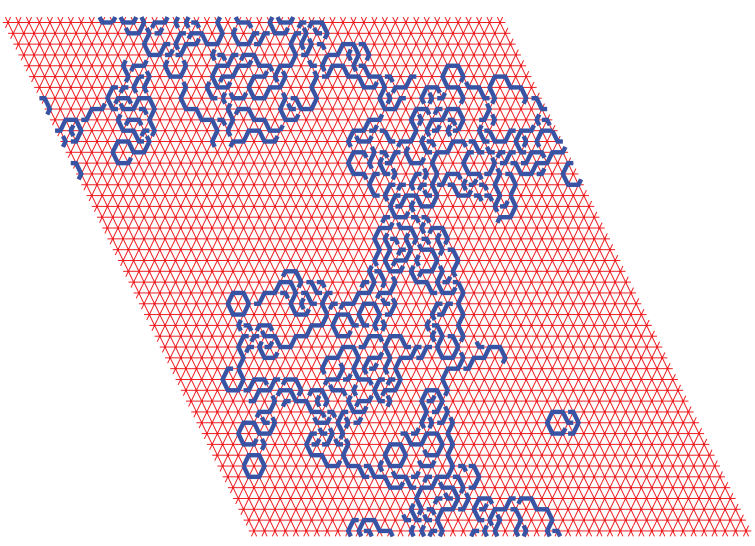

FIG. 7. (Color online) Configuration for the $m$-2A4B model with $y=0.44, \rho=1 / 3, T^{*}=0.10$. At these conditions there is LVE phase separation.

the reentrant solubility at low temperature is attributed to the effects of large anisotropic interactions (hydrogen bonding) between the two components, whereas the demixing at intermediate temperatures is due to van der Waals interactions, which favor the interaction between pairs of particles of the same species [29,30].

The results presented in this Rapid Communication resemble, to some extent, those reported by Sciortino et al. [31,32] for a system of Janus particles, and by Reinhardt et al. [33] for systems with competition between the phase separation and self-assembly. In both cases, at low temperature, the vapor density at LVE increases on cooling. However, in these systems no LCP appears, due to the presence of crystalline phases that make both liquid and vapor metastable prior to the possible presence of the LCP.

The finding of closed-loop LVE in the $m$-2A4B model suggests the likelihood of finding the same behavior for related patchy models in the continuum for both two- and three-dimensional models. We are currently working in this direction.

As a summary, we have shown how patchy models on the triangular lattice can exhibit closed-loop LVE on one-component systems. The basic physics underlying this behavior is the reduction of valence at low temperature by the formation of compact structures at low and moderate densities that reduce the amount of connectivity between different parts of the systems. Finally, self-assembling mechanisms similar to those appearing in the simple models presented in this Rapid Communication might play some role in the reentrant condensation phenomena of globular proteins [8].

The author gratefully acknowledges support from the Dirección General de Investigación Científica y Técnica under Grant No. FIS2010-15502, and from the Dirección General de Universidades e Investigación de la Comunidad de Madrid under Grant No. S2009/ESP-1691 and Program MODELICOCM.
[1] A. B. Pawar and I. Kretzschmar, Macromol. Rapid Commun. 31, 150 (2010).

[2] F. Sciortino and E. Zaccarelli, Curr. Opin. Solid State Mater. Sci. 15, 246 (2011).

[3] S. C. Glotzer and M. J. Solomon, Nat. Mater. 6, 557 (2007).

[4] E. Bianchi, R. Blaak, and C. N. Likos, Phys. Chem. Chem. Phys. 13, 6397 (2011).

[5] F. Zhang et al., Phys. Rev. Lett. 101, 148101 (2008).

[6] F. Zhang et al., Proteins 78, 3450 (2010).

[7] R. Piazza, Curr. Opin. Colloid Interface Sci. 8, 515 (2004).

[8] F. Zhang et al., Faraday Discuss. (2012), doi: 10.1039/C2FD20021J.

[9] J. Russo, J. M. Tavares, P. I. C. Teixeira, M. M. Telo da Gama, and F. Sciortino, J. Chem. Phys. 135, 034501 (2011).

[10] J. Russo, J. M. Tavares, P. I. C. Teixeira, M. M. Telo da Gama, and F. Sciortino, Phys. Rev. Lett. 106, 085703 (2011).

[11] N. G. Almarza, J. M. Tavares, M. Simões, and M. M. Telo da Gama, J. Chem. Phys. 135, 174903 (2011).

[12] J. M. Tavares, P. I. C. Teixeira, and M. M. Telo da Gama, Phys. Rev. E 80, 021506 (2009).

[13] A. Giacometti, F. Lado, J. Largo, G. Pastore, and F. Sciortino, J. Chem. Phys. 132, 174110 (2010).

[14] N. G. Almarza, J. M. Tavares, and M. M. Telo da Gama, Phys. Rev. E 82, 061117 (2010).

[15] N. G. Almarza, J. M. Tavares, and M. M. Telo da Gama, J. Chem. Phys. 134, 071101 (2011)

[16] N. G. Almarza, J. M. Tavares, and M. M. Telo da Gama, Phys. Rev. E 85, 053102 (2012).
[17] N. G. Almarza and E. G. Noya, Mol. Phys. 109, 65 (2011).

[18] J. S. Høye, E. Lomba, and N. G. Almarza, Mol. Phys. 107, 321 (2009).

[19] N. G. Almarza, J. Capitan, J. Cuesta, and E. Lomba, J. Chem. Phys. 131, 124506 (2009).

[20] C. J. Roberts, A. Z. Panagiotopoulos, and P. G. Debenedetti, Phys. Rev. Lett. 77, 4386 (1996).

[21] M. M. Szortyka, C. E. Fiore, V. B. Henriques, and M. C. Barbosa, J. Chem. Phys. 133, 104904 (2010).

[22] M. Szortyka, M. Girardi, V. B. Henriques, and M. C. Barbosa, J. Chem. Phys. 132, 134904 (2010).

[23] M. Szortyka, M. Girardi, V. B. Henriques, and M. C. Barbosa, J. Chem. Phys. 130, 184902 (2009).

[24] G. I. Menon, R. Pandit, and M. Barma, Europhys. Lett. 24, 253 (1993).

[25] G. I. Menon and R. Pandit, Phys. Rev. E 59, 787 (1999).

[26] E. Lomba, C. Martín, N. G. Almarza, and F. Lado, Phys. Rev. E 71, 046132 (2005).

[27] N. G. Almarza (unpublished).

[28] J. M. Tavares, L. Rovigatti, and F. Sciortino, J. Chem. Phys. 137, 044901 (2012).

[29] J. Hirschfelder, D. Stevenson, and H. Eyring, J. Chem. Phys. 5, 896 (1937).

[30] R. E. Goldstein, J. Chem. Phys. 83, 1246 (1985).

[31] F. Sciortino, A. Giacometti, and G. Pastore, Phys. Chem. Chem. Phys. 12, 11869 (2010).

[32] F. Sciortino, A. Giacometti, and G. Pastore, Phys. Rev. Lett. 103, 237801 (2009).

[33] A. Reinhardt et al., J. Chem. Phys. 134, 104905 (2011). 